\title{
EFFECT OF TETRATHIAFULVALENE- TETRACYANOQUINODIMETHANE ADDITION ON THE MECHANICAL PROPERTIES OF POLYAMIDE 6 NANOFIBERS
}

\author{
KAZUTO TANAKA, NANAMI YAMAMURO \& TSUTAO KATAYAMA \\ Department of Biomedical Engineering, Doshisha University, Japan
}

\begin{abstract}
Tetrathiafulvalene-tetracyanoquinodimethane (TTF-TCNQ) is an organic semiconductor with excellent mechanical flexibility and it is attracting attention as a material of bendable devices. Nanofibers containing TTF-TCNQ can be used as sensors and electrodes. In order to apply nanofiber nonwoven fabrics with TTF-TCNQ into practical use of a wide range of fields utilizing its mechanical flexibility, it is necessary to clarify the effect of the additive amount of TTF-TCNQ on the mechanical properties of nanofiber nonwoven fabrics. In this study, TTF-TCNQ/Polyamide 6 (PA6) nanofiber nonwoven fabric was fabricated by electrospinning method, and the effect of the additive amount of TTF-TCNQ on the mechanical properties of nanofiber nonwoven fabric was revealed by the tensile test. The tensile strength of PA6 nanofiber with 1 wt.\% TTF-TCNQ was larger than that of nanofiber without TTF-TCNQ, and it fractured in a ductile manner. On the other hand, tensile strength of PA6 nanofiber with $10 \mathrm{wt} . \%$ TTF-TCNQ was smaller because of the aggregation of TTF-TCNQ.

Keywords: TTF-TCNQ/PA6 nanofiber nonwoven fabric, electrospinning, tensile strength, fracture behavior, volume resistivity.
\end{abstract}

\section{INTRODUCTION}

Tetrathiafulvalene-tetracyanoquinodimethane (TTF-TCNQ) is an organic semiconductor which has the same electrical conductivity as metal and is excellent in mechanical flexibility [1], [2]. It is attracting attention as a material of bendable organic electroluminescent devices and as organic field effect transistors [3], [4]. Nanofibers have a large specific surface area, high specific strength, and high specific rigidity; therefore, they are being used in various fields such as biotechnology, environment and IT. Conductivity is one of the promising properties to be added to nanofibers for these applications. In order to fabricate conductive nanofibers, some techniques to use conductive polymers and to plate metal on nanofiber surfaces have been used. Conductive polymers are electrically unstable while metal-plated nanofibers are high in electrical conductivity. However, metal-plated nanofibers have problems with metals peeling off when deformed, which reduce their electrical performance significantly [5]-[7].

Nanofibers with conductive fine particles are attracting attention as a material to overcome these problems, therefore, nanofibers containing TTF-TCNQ are expected to be used as sensors and electrodes. In order to apply nanofiber nonwoven fabrics with TTF-TCNQ into practical use at a wide range of fields utilizing its mechanical flexibility, it is necessary to clarify the effect of the additive amount of TTF-TCNQ on the mechanical properties of nanofiber nonwoven fabrics.

In this study, TTF-TCNQ/PA6 nanofiber nonwoven fabric was fabricated by electrospinning method. Polyamide 6 (PA6), which has excellent wear resistance and impact resistance, was used as the base material of nanofiber, and synthesized TTF-TCNQ was added to PA6. The effect of the additive amount of TTF-TCNQ on the mechanical properties 
of TTF-TCNQ/PA6 nanofiber nonwoven fabric was revealed by the tensile test and volume resistance measurement.

\section{MATERIALS AND EXPERIMENTAL PROCEDURES}

\subsection{Synthesis of TTF-TCNQ and preparation of TTF-TCNQ/PA6 nanofibers}

After TTF $0.1 \mathrm{~g}$ and TCNQ $0.1 \mathrm{~g}$ were dissolving at $80^{\circ} \mathrm{C}$ in $10 \mathrm{ml}$ and $15 \mathrm{ml}$ of acetonitrile respectively, TTF-TCNQ is synthesized by quenching their mixed solution [8]. Synthesized TTF-TCNQ was dissolved in formic acid under $28 \mathrm{kHz}$ of ultrasonic stirring for 10 minutes. PA6 resin pellet (1015B, Ube Industries, Ltd.) as a polymer material and Dichloromethane (DCM, NACALAI TESQUE, INC.) were added into the formic acid/TTF-TCNQ solution, and dissolved by stirring for 1 hour 30 minutes to prepare a polymer solution. The weight ratio of formic acid and DCM was 1:1 [9], and the amount of TTF-TCNQ was $1 \mathrm{wt} . \%$ and 10 wt.\% based on PA6. Nanofiber nonwoven fabrics were spun for 30 minutes by the electrospinning unit (NANON, MECC; Fig. 1) using the prepared solution. TTF-TCNQ/PA6 nanofiber nonwoven fabrics with $1 \mathrm{wt} . \%$ and $10 \mathrm{wt} . \%$ TTF-TCNQ are referred to as TTF- TCNQ/PA6 (1 wt.\%) and TTF-TCNQ/PA6 (10 wt.\%), respectively. The spinning conditions were as follows: applied voltage $25 \mathrm{kV}$, spinning distance: $150 \mathrm{~mm}$, syringe feed rate: $1 \mathrm{ml} / \mathrm{h}$, and nanofibers were spun on aluminum foil placed on a flat plate collector. The inside condition of the electrospinning unit was controlled as $23 \pm 4^{\circ} \mathrm{C}, 35 \pm 5 \% \mathrm{RH}$. The spun nanofiber nonwoven fabric was observed with a digital microscope (VHX-5000, KEYENCE) and a scanning electron microscope (SEM, JSM-6390LT, JEOL).
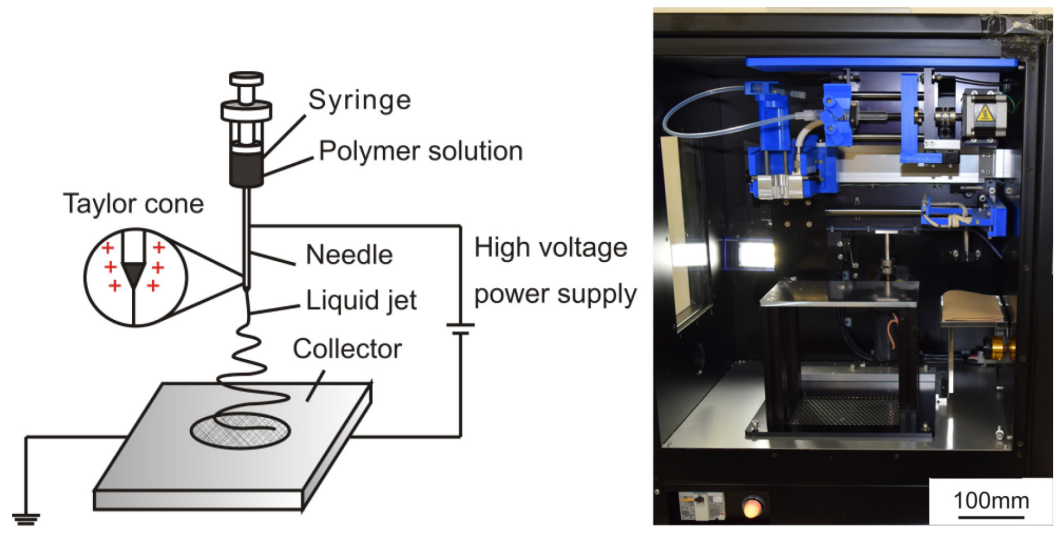

Figure 1: Schematic drawing and photo of electrospinning unit.

\subsection{Tensile test}

After attaching a PET film tab to the nanofiber nonwoven fabric which was spun onto the aluminum foil, it was punched into a dumbbell shape by a punching machine, and fixed with another PET film tab to prepare the test specimen as shown in Fig. 2. For the tensile test, a small desktop tester (EZ-graph, Shimadzu Co., Japan) equipped with $5 \mathrm{~N}$ load cells was used at a displacement speed of $1 \mathrm{~mm} / \mathrm{min}$. The parallel length and the width of the test specimen was $16 \mathrm{~mm}$ and $2 \mathrm{~mm}$, respectively. After attaching the test specimen to the jig, the tab of the test specimen shown in Fig. 2 was cut off, and the test was performed so that the load was 
applied only to the nanofiber nonwoven fabric. A micrometer (MITUTOYO) was used to measure the thickness of the specimen, and a digital microscope (VHX-5000, KEYENCE) was used to measure width of the specimen. In this study, the nominal stress was defined as the value obtained by dividing the load by the initial cross-sectional area calculated from the measured thickness and the width at the center of the specimen. For the observation by scanning electron microscope (SEM, JSM-6390LT, JEOL), the surface was coated with osmium of $40 \mathrm{~nm}$ using an osmium plasma coater (OPC60A, Filgen).

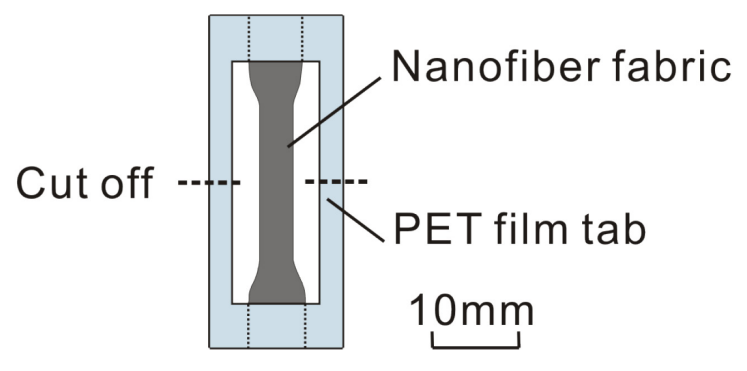

Figure 2: Schematic drawing of tensile test specimen.

\subsection{Measurement of volume resistivity}

As shown in Fig. 3, the volume resistance of the TTF-TCNQ/PA6 nanofiber nonwoven fabric was measured using a super insulation meter (SM7120, HIOKI) with a nonwoven fabric sandwiched between two copper plates with load of $30 \mathrm{~N}$. Volume resistivity $\left(\rho_{v}\right)$ was calculated using the measured volume resistance value $(\Omega)$ and the following eqn (1). In the following equation, $S$ is the cross-sectional area of copper plate, and $t$ is the thickness of the nanofiber nonwoven fabric

$$
\rho_{v}=\Omega \frac{S}{t}
$$

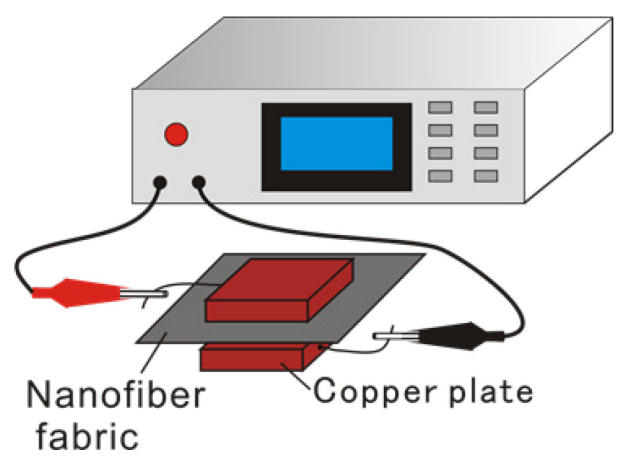

Figure 3: Schematic drawing of apparatus for measuring volume resistivity.

\section{RESULTS AND DISCUSSION}

Fig. 4 shows an example of the digital microscope images of TTF-TCNQ/PA6 (1 wt.\%) and TTF-TCNQ/PA6 (10 wt.\%). In the case of TTF-TCNQ/PA6 (1 wt.\%), TTF-TCNQ was well 
dispersed, and aggregated black part was not observed. On the other hand, some TTF-TCNQ aggregates were observed in TTF-TCNQ/PA6 (10 wt.\%).

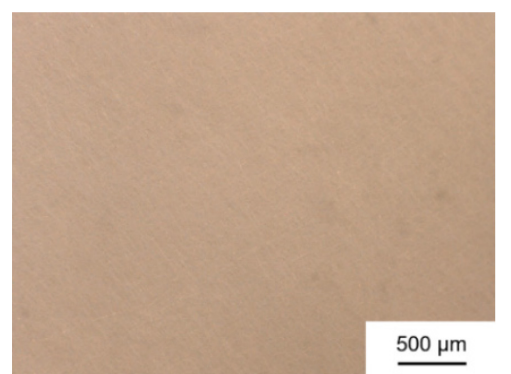

(a)

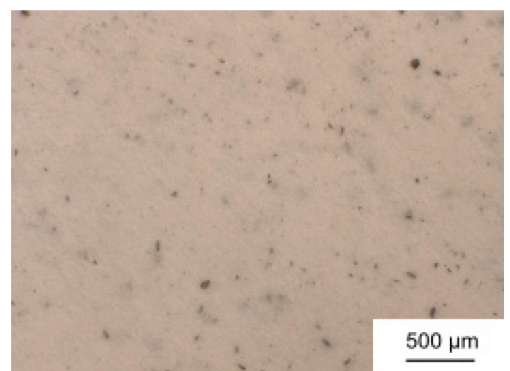

(b)

Figure 4: Images of TTF-TCNQ/PA6 random fabric. (a) TTF-TCNQ/PA6 (1 wt.\%); (b) TTF-TCNQ/PA6 (10 wt.\%).

\subsection{Tensile test}

The stress-displacement curves and the obtained tensile strength of TTF-TCNQ/PA6 nanofiber nonwoven fabric with different additive amounts of TTF-TCNQ, and PA6 nanofiber nonwoven fabric are shown in Figs 5 and 6, respectively. In Fig. 5, after the liner deformation with high gradient, the gradient changed to the lower value. TTF-TCNQ/PA6 (1 wt.\%), in which TTF-TCNQ was well dispersed, has the highest tensile strength among the three conditions. On the other hand, the tensile strength of TTF-TCNQ/PA6 (10 wt.\%) was lower than that of the PA6 nanofiber nonwoven fabric. In the case of large additive amount of TTF-TCNQ, aggregated TTF-TCNQ as shown in Fig. 4, is considered to be the crack initiation and to show the lower tensile strength.

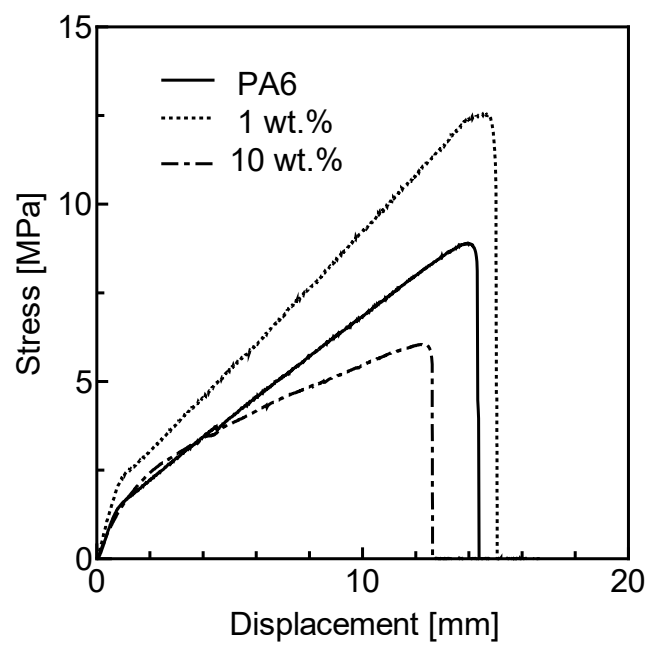

Figure 5: Stress-displacement curve of PA6, TTF-TCNQ/PA6 nanofiber nonwoven fabric. $(\mathrm{N}=3$, mean \pm S.D.). 


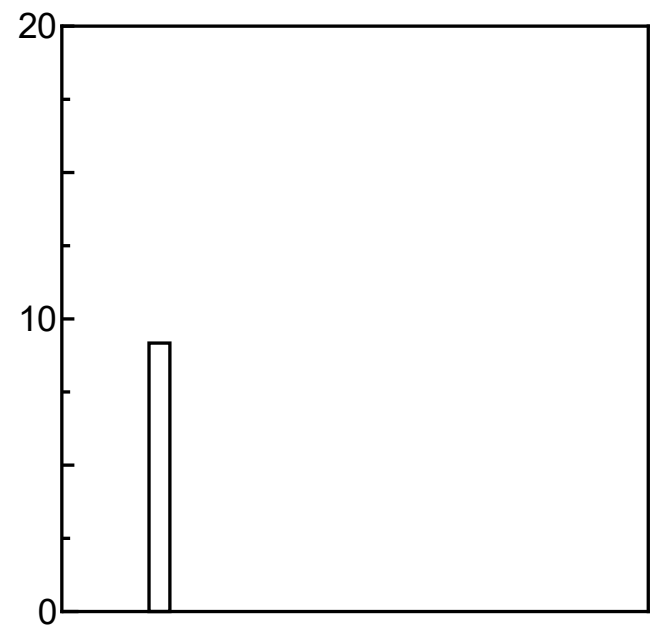

Figure 6: Tensile strengths of PA6 and TTF-TCNQ/PA6 nanofiber nonwoven fabric. $(\mathrm{N}=3$, mean \pm S.D.).

Fig. 7 shows the SEM images of the TTF-TCNQ/PA6 nanofiber nonwoven fabric after tensile test. In the case of TTF-TCNQ/PA6 ( $1 \mathrm{wt} . \%)$, the fibers were aligned to the longitudinal direction by tensile load and it fractured in a ductile manner. The PA6 nanofiber nonwoven fabric also showed similar fracture as TTF-TCNQ/PA6 (1 wt.\%) On the other hand, for TTF-TCNQ/PA6 (10 wt.\%), the alignment of the fibers in the longitudinal direction was relatively small, and it fractured in a brittle manner.

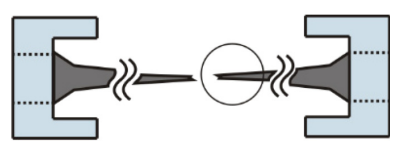

Observed area

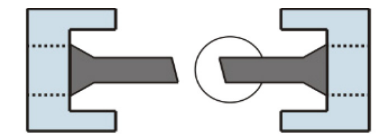

Observed area

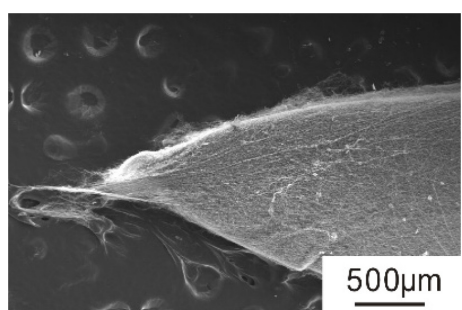

(a)

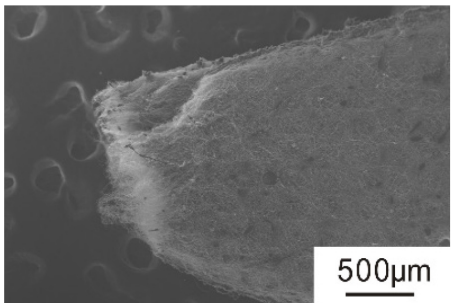

(b)

Figure 7: SEM observation of the nonwoven fabric after the tensile test. (a) TTF-TCNQ/PA6 (1 wt.\%); (b) TTF-TCNQ/PA6 (10 wt.\%). 


\subsection{Measurement of volume resistivity}

Fig. 8 shows the results of the volume resistivity of TTF-TCNQ/PA6 nanofiber nonwoven fabrics with different additive amounts of TTF-TCNQ. The volume resistivity of PA6 nanofibers, TCNQ/PA6 (1 wt.\%) and TCNQ/PA6 (10 wt.\%) was $8.10 \mathrm{~T} \Omega \cdot \mathrm{m}, 5.93 \mathrm{~T} \Omega \cdot \mathrm{m}$ and $1.18 \mathrm{~T} \Omega \cdot \mathrm{m}$, respectively. The volume resistivity of TCNQ/PA6 (10 wt.\%) is $85 \%$ lower than that of PA6 nanofiber. Resistivity is considered to be decreased by the increase of contact probability between TTF-TCNQs with the increase of the additive amount.

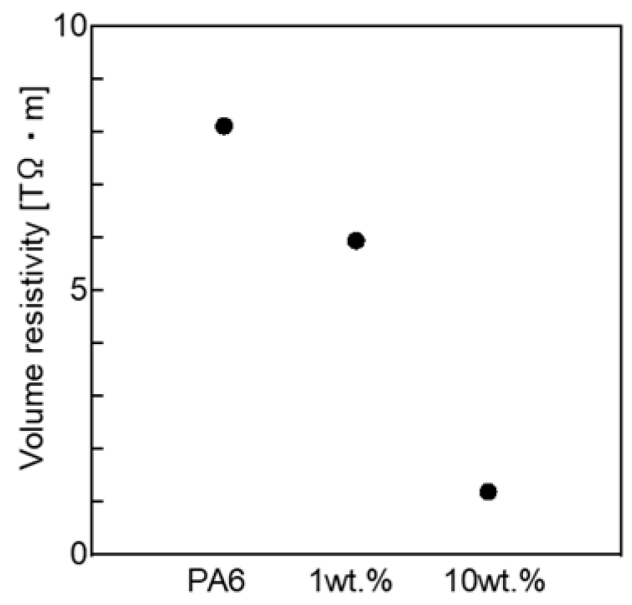

Figure 8: Volume resistivity in case of adding TTF-TCNQ.

\section{CONCLUSION}

Using the synthesized TTF-TCNQ, TTF-TCNQ/PA6 nanofiber nonwoven fabrics were fabricated by electrospinning method, and tensile test and volume resistance measurement were conducted to reveal the effect of the additive amount of TTF-TCNQ on the mechanical properties of nanofiber nonwoven fabric. The investigation yielded the following conclusions:

1. The addition of TTF-TCNQ can lower the volume resistivity of PA6 nanofiber nonwoven fabrics. PA6 nanofiber nonwoven fabrics with $10 \mathrm{wt} \%$ of TTF-TCNQ has the volume resistivity of $1.18 \mathrm{~T} \Omega \cdot \mathrm{m}$, although PA6 nanofiber nonwoven fabrics has $8.10 \mathrm{~T} \Omega \cdot \mathrm{m}$.

2. PA6 nanofiber nonwoven fabrics with 1 wt. $\%$ of TTF-TCNQ has the highest tensile strength.

\section{REFERENCES}

[1] Thomas, G.A., Schafer, D.E. \& Wudl, F., Electrical conductivity of tetrathiafulvalenium-tetracyanoquinodimethanide (TTF-TCNQ). Physical Reviewed, pp. 5105-5110, 1976.

[2] Okada, H., Tanaka, K. \& Chujo, Y., Preparation of environmentally resistant conductive silica-based polymer hybrids containing tetrathiafulvalene-tetracyanoquinodimethane charge-transfer complexes. Polymer Journal, 46, pp. 800-805, 2014. 
[3] Sakai, M., Sakuma, H., Ito, Y., Saito, A., Nakamura, M. \& Kubo, K., Ambipolar fieldeffect transistor characteristics of (BEDT-TTF)(TCNQ) crystals and metal-like conduction induced by a gate electric field. The American Physical Society, 76, 045111, 2007.

[4] Georgakopoulos, S. et al., Spray-coated contacts from an organic charge transfer complex solution for organic field-effect. Organic Electronics, 48, pp. 365-370, 2017.

[5] Zhang, N. et al., Lightweight and flexible Ni-Co alloy nanoparticle-coated electrospun polymer nanofiber hybrid membranes for high performance electromagnetic interference shielding. Journal of Alloys and Compounds, 784, pp. 244-255, 2019.

[6] Marx, S., Jose, M., Andersen, J. \& Russell, A., Electrospun gold nanofiber electrodes for biosensors. Biosensors and Bioelectronics, 26, pp. 2981-2986, 2011.

[7] Park, J. et al., Electromagnetic interference shielding effectiveness of sputtered $\mathrm{NiFe} / \mathrm{Cu}$ multi-layer thin film at high frequencies. Thin Solid Films, 677, pp. 130-136, 2019.

[8] http://mms.ltm.kyoto-u.ac.jp/yamochi/jpn/index.html.

[9] Tanaka, K., Hashimoto, M. \& Katayama, T., Development of Fabrication Process of Carbon Nanotube Reinforced Polyamide 6 (PA6) Nanofiber and Evaluation of its Mechanical Properties, The Society of Materials Science, Japan, 2015. 